\title{
Magnetoelectric effect due to local noncentrosymmetry
}

\author{
V P Sakhnenko and N V Ter-Oganessian \\ Institute of Physics, Southern Federal University, 194 Stachki Prospekt, \\ Rostov-on-Don, 344090 Russia \\ E-mail: nikita.teroganessian@gmail.com
}

\begin{abstract}
Magnetoelectrics often possess ions located in noncentrosymmetric surroundings. Based on this fact we suggest a microscopic model of magnetoelectric interaction and show that the spin-orbit coupling leads to spin-dependent electric dipole moments of the electron orbitals of these ions, which results in non-vanishing polarization for certain spin configurations. The approach accounts for the macroscopic symmetry of the unit cell and is valid both for commensurate and complex incommensurate magnetic structures. The model is illustrated by the examples of $\mathrm{MnWO}_{4}, \mathrm{MnPS}_{3}$ and $\mathrm{LiNiPO}_{4}$. Application to other magnetoelectrics is discussed.
\end{abstract}

PACS numbers: 75.85.+t, 77.84.-s, 71.70.Ej 


\section{Introduction}

Pierre Curie [1] was the first to predict the interplay between magnetism and ferroelectricity. The macroscopic symmetry consideration of the magnetoelectric (ME) effect was given by Dzyaloshinskii only in 1959 [2], whereas experimentally it was discovered by Astrov in $\mathrm{Cr}_{2} \mathrm{O}_{3}$ in 1960 [3]. In the last decade whole new classes of magnetoelectric materials were discovered and experimentally studied (for a recent review see, for example, [4]).

Despite a long history of ME effect its microscopic origin is still a subject of debate. The fact that ferroelectricity commonly occurs in incommensurately modulated magnetically ordered phases predominates the assumption and consideration of various complex types of magnetic order such as screw, cycloidal, helix and others in most of the microscopic models of magnetoelectricity [4, 5]. From the macroscopic crystal symmetry point of view the close connection between the appearance of modulated and ferroelectric phases in magnetoelectrics was recently pointed out [6]. Whereas electric polarization indeed often occurs concurrently with complex modulated spin structures it can also be induced by commensurate magnetic order (such is the case, for example, in some rare-earth manganates $\mathrm{RMn}_{2} \mathrm{O}_{5}[7]$ ).

Currently two models of ME coupling are widely accepted in literature. In the model by Sergienko et al. [8] electric polarization $\vec{P} \sim\left[\vec{S}_{i} \times \vec{S}_{i+1}\right]$ is induced by the Dzyaloshinskii-Moriya (DM) interaction between two magnetic ions $i$ and $i+1$ with superexchange. In the spin current model by Katsura et al. 9] the electric polarization is due to the spin supercurrent $\vec{P} \sim \vec{e}_{i j} \times\left[\vec{S}_{i} \times \vec{S}_{j}\right]$ with $\vec{e}_{i j}$ being the unit vector connecting the sites $i$ and $j$. Interpretation of the experimentally observed polarization using these models meets difficulties though and was a subject of critique [10, 11]. It was argued [11] that the DM coupling [8] is 2 orders of magnitude weaker than what is needed to explain experimental situation in magnetoelectric manganites. On the other hand in the spin current model 9 the authors also overestimated up to 2 orders of magnitude the value of the magnetically induced polarization [11]. Several other mechanisms of the ME effect were proposed [11, 12] but as argued by the authors themselves they are insufficient to explain magnetoelectricity in some $\mathrm{Cu}^{2+}$ magnetoelectrics.

From our point of view most of the microscopic models proposed so far do not take into account the macroscopic crystal symmetry mainly focusing on three-site clusters (two metal ions and oxygen) and considering various spiral magnetic structures. The authors of the DM model of ME effect [8], for example, apply it to the case of rareearth manganites $\mathrm{RMnO}_{3}$, whereas it can be shown that the macroscopic polarization vanishes when one considers the orthorhombic symmetry of the unit cell. Indeed, the authors take $\mathrm{TbMnO}_{3}$ as an example using the spiral magnetic structure available from neutron diffraction data. They consider the polarization induced in the $x$ and $y$ chains of Mn-O-Mn, but erroneously take into account only the $z=0$ plane. Indeed, when one considers also the $z=1 / 2$ plane (obtained from the first one by $\sigma_{z}\left(00 \frac{1}{2}\right)$ ) the macroscopic polarization cancels out. 

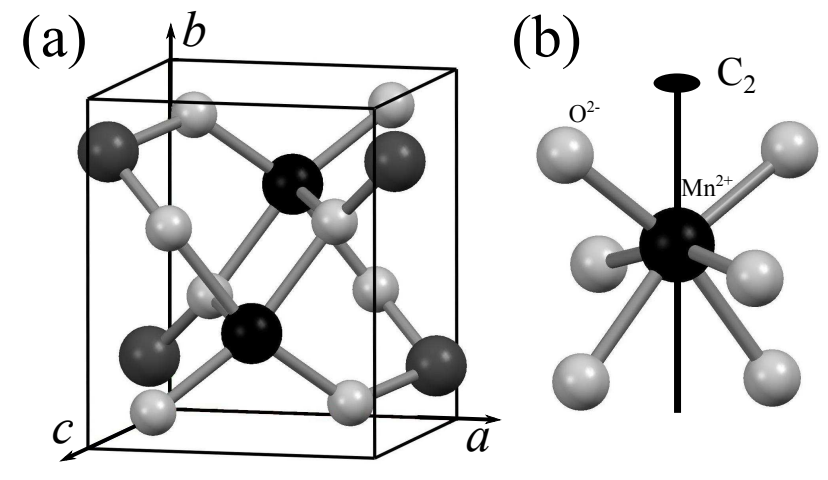

Figure 1. (a) The monoclinic unit cell of $\mathrm{MnWO}_{4} \cdot \mathrm{Mn}^{2+}, \mathrm{W}^{6+}$ and $\mathrm{O}^{2-}$ ions are shown by black, dark gray and light gray circles, respectively. (b) The $\mathrm{Mn}^{2+}$ cluster showing distorted oxygen octahedron. The rotational $\mathrm{C}_{2}$ symmetry axis is indicated.

The aim of any ME model is to find the mechanism of inversion symmetry breaking by magnetic order. At the same time some authors explicitly start with a centrosymmetric cluster such as, for example, in the spin current [9, 13] or the $\mathrm{MeO}_{n}$ cluster model [11]. This dismisses the fact that in many magnetoelectrics some types of ions are located in noncentrosymmetric surroundings already in the paramagnetic phase. In this work using $\mathrm{MnWO}_{4}$ as an example we suggest a microscopic magnetoelectric coupling model taking into account macroscopic symmetry of the unit cell and noncentrosymmetric surrounding of the $\mathrm{Mn}^{2+}$ ions. We then apply our microscopic model to estimate the linear magnetoelectric coefficients in $\mathrm{MnPS}_{3}$ and $\mathrm{LiNiPO}_{4}$ and discuss its application to other magnetoelectrics.

\section{Microscopic model}

Wolframite $\mathrm{MnWO}_{4}$ possesses a monoclinic structure at room temperature (figure 1a) described by the space group $P 2 / c\left(\mathrm{C}_{2 h}^{4}\right)$. On lowering the temperature it undergoes a sequence of magnetic phase transitions at $13.5 \mathrm{~K}\left(\mathrm{~T}_{N}\right), 12.7 \mathrm{~K}\left(\mathrm{~T}_{2}\right)$ and $7.6 \mathrm{~K}$ $\left(\mathrm{T}_{1}\right)$, leading to the appearance of magnetically ordered states AF3, AF2 and AF1 [14, respectively. The low temperature phase AF1 is characterized by the wave vector $\vec{k}=(1 / 4 ; 1 / 2 ; 1 / 2)$, whereas the incommensurate phases AF2 and AF3 by $(-0.214 ; 1 / 2 ; 0.457)$. Electric polarization in $\mathrm{MnWO}_{4}$ appears in the AF2 phase along the crystal $b$ axis [15]. The phenomenological model of phase transitions in wolframite was suggested earlier based on the assumption that the magnetic order is driven by the instability in the $(1 / 4 ; 1 / 2 ; 1 / 2)$ point of the Brillouin zone [16]. However, for our purpose of building a microscopic model of the ME effect we first start with a hypothetical magnetic order with $\vec{k}=0$ in $\mathrm{MnWO}_{4}$ (i. e. without multiplication of the unit cell) and then consider the real magnetic structure.

$\mathrm{MnWO}_{4}$ contains two magnetic $\mathrm{Mn}^{2+}$ ions $\mathrm{Mn}_{1}$ and $\mathrm{Mn}_{2}$ in the unit cell located at positions $(0.5 ; 0.6853 ; 0.25)$ and $(0.5 ; 0.3147 ; 0.75)$, respectively. In the following we define the orthogonal $x, y$ and $z$ axes parallel to the $a$ axis, parallel to the $b$ axis 
Table 1. IR's of the $P 2 / c$ space group corresponding to $\vec{k}=0$. The last column lists the components of $\vec{F}, \vec{A}$ and $\vec{P}$ according to the IR's upon which they transform. Note that $\vec{F}$ and $\vec{A}$ are odd under time inversion.

\begin{tabular}{lccl}
\hline $\mathrm{IR}$ & $C_{2 y}\left(00 \frac{1}{2}\right)$ & $I(000)$ & Order parameters \\
\hline $\mathrm{GM}^{1+}$ & 1 & 1 & $F_{y}$ \\
$\mathrm{GM}^{1-}$ & 1 & -1 & $P_{y}, A_{y}$ \\
$\mathrm{GM}^{2+}$ & -1 & 1 & $F_{x}, F_{z}$ \\
$\mathrm{GM}^{2-}$ & -1 & -1 & $P_{x}, P_{z}, A_{x}, A_{z}$ \\
\hline
\end{tabular}

and perpendicular to both the $a$ and $b$ axes of the monoclinic cell, respectively. For the description of the magnetic order with $\vec{k}=0$ we can introduce ferromagnetic and antiferromagnetic order parameters $\vec{F}=\vec{S}_{1}+\vec{S}_{2}$ and $\vec{A}=\vec{S}_{1}-\vec{S}_{2}$, respectively, where $\vec{S}_{1}$ and $\vec{S}_{2}$ are the magnetic moments of $\mathrm{Mn}_{1}$ and $\mathrm{Mn}_{2}$. Table 1 summarizes the irreducible representations (IR) possessed by the $P 2 / c$ space group in the center of the Brillouin zone and shows how the components of $\vec{F}, \vec{A}$ and electric polarization $\vec{P}$ transform according to the symmetry operations of the group. As evident from the table the following magnetoelectric interactions are allowed by the macroscopic symmetry

$$
\begin{aligned}
& P_{\mu} A_{x} F_{y}, \quad P_{\mu} A_{y} F_{x}, \quad P_{\mu} A_{y} F_{z}, \quad P_{\mu} A_{z} F_{y}, \\
& P_{y} A_{\alpha} F_{\alpha}, \quad P_{y} A_{x} F_{z}, \quad P_{y} A_{z} F_{x},
\end{aligned}
$$

where $\mu=x, z$ and $\alpha=x, y, z$. Thus any combination of ferromagnetic and antiferromagnetic ordering produces electric polarization. The polarization also arises in case when only one of the $\mathrm{Mn}^{2+}$ moments orders (e. g. when $\vec{S}_{1} \neq 0$ and $\vec{S}_{2}=0$, which corresponds to $\vec{F}=\vec{A}$ ). Such collinear commensurate magnetic ordering gives no polarization according to any of the microscopic models proposed so far, since they are usually concentrated on noncollinear modulated magnetic structures. Nevertheless, from the crystal symmetry point of view such magnetic order breaks the crystallographic equivalency of $\mathrm{Mn}_{1}$ and $\mathrm{Mn}_{2}$ atoms, which are connected by inversion (i. e. breaks inversion symmetry) and electric polarization arises.

In order to build a valid microscopic model of $\mathrm{ME}$ interaction we proceed with the following consideration. First we note that the $\mathrm{Mn}^{2+}$ ions in $\mathrm{MnWO}_{4}$ are located in noncentrosymmetric positions. This can be seen from the fact that $I(000)$ interchanges $\mathrm{Mn}_{1}$ and $\mathrm{Mn}_{2}$ or by direct examination of oxygen positions around $\mathrm{Mn}^{2+}$ ions [14]. Since $C_{2 y}\left(00 \frac{1}{2}\right)$ transforms each $\mathrm{Mn}^{2+}$ ion into itself their local crystal symmetry is $\mathrm{C}_{2}$ as depicted in figure 1b. Thus the manganese ions are located in polar surroundings and all their electron's orbitals have electric dipole moment along the $y$ axis. Every monoclinic cell has two $\mathrm{Mn}^{2+}$ ions with opposite dipole moments, thus, preserving zero dipole moment of the unit cell as schematically shown in figure 2a.

Figure $2 \mathrm{~b}$ shows an ion in polar crystal environment (local symmetry $\mathrm{C}_{2}$ ). All electron orbitals possess, therefore, an electric dipole moment. If one takes the spin degree of freedom into account then the spin-orbit coupling alters the electric dipole moment. When the spin directs away from the two-fold rotational axis it breaks the 


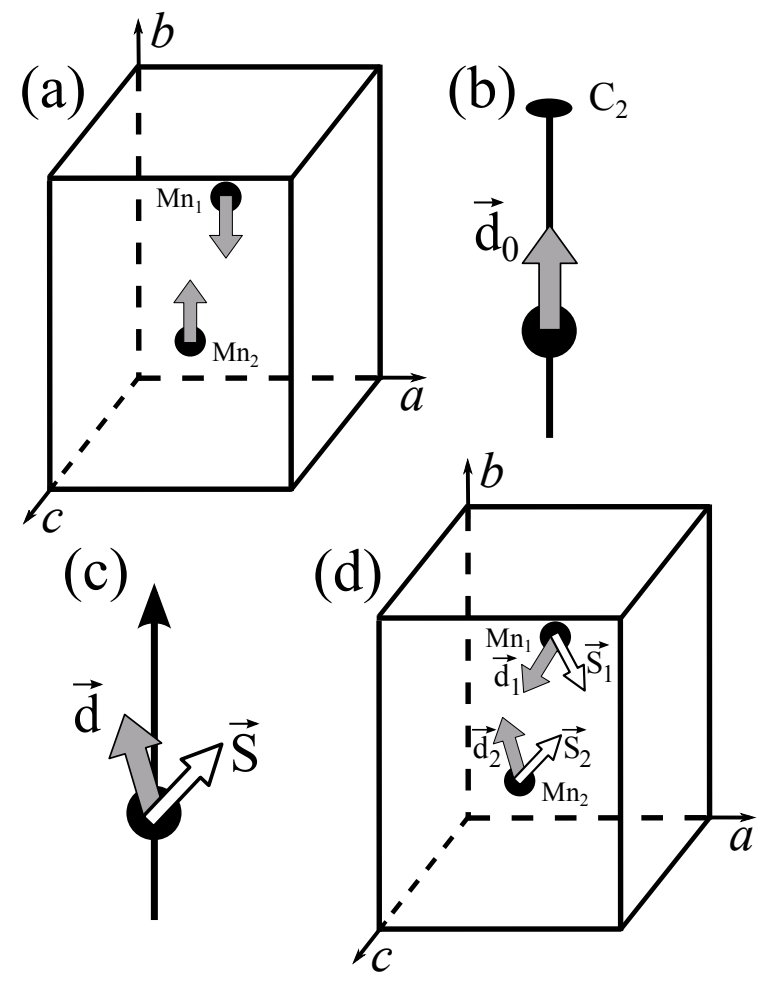

Figure 2. (a) Two $\mathrm{Mn}^{2+}$ ions in the monoclinic unit cell with opposing electric dipole moments (gray arrows). (b) An ion (black circle) in $\mathrm{C}_{2}$ crystal field (indicated) possessing dipole moment $\vec{d}_{0}$ (gray arrow). (c) Same as (b) but taking the spin degree of freedom into account. A spin $\vec{S}$ (white arrow) deviating from the $\mathrm{C}_{2}$ axis breaks the rotational symmetry and changes the electric dipole moment to $\vec{d}$ deflecting it from the initial symmetry axis. (d) Two $\mathrm{Mn}^{2+}$ ions in the monoclinic unit cell with spin dependent electric dipole moments $\vec{d}_{1}$ and $\vec{d}_{2}$ resulting in nonzero total dipole moment of the unit cell $\vec{d}_{1}+\vec{d}_{2}$.

rotational symmetry resulting in modification of the dipole moment as schematically shown in figure 2k. We now proceed from this simple physics consideration to semiquantitative quantum mechanical treatment.

The manganese ions in $\mathrm{MnWO}_{4}$ are located in distorted oxygen octahedra as shown in figure 1 $1 \mathrm{~b}$. Therefore we start with a $\mathrm{Mn}^{2+}$ ion in octahedral crystal field and for simplicity consider only the $e_{g}$ orbitals. Inclusion of $t_{2 g}$ levels is straightforward. Thus as a zeroth order perturbation we have

$$
H_{0}\left|d_{\gamma}\right\rangle=E_{d}\left|d_{\gamma}\right\rangle
$$

where $\gamma=z^{2}$ or $x^{2}-y^{2}, H_{0}$ is the hamiltonian including octahedral splitting field and $E_{d}$ is the $e_{g}$ energy level. Next, we include as perturbation the monoclinic crystal field of $\mathrm{C}_{2}$ symmetry assuming the polar axis along the $z$ axis

$$
V_{C F}=c_{z} z+c_{x y} x y+c_{x y}^{\prime}\left(x^{2}-y^{2}\right)+c_{z}^{\prime} z^{2}
$$

and spin-orbit coupling

$$
V_{S O}=-\lambda(\vec{L} \cdot \vec{S})
$$


Here $c_{z}, c_{x y}, c_{x y}^{\prime}$ and $c_{z}^{\prime}$ are coefficients, $\vec{L}$ is the angular momentum operator, $\vec{S}$ is the spin and $\lambda$ is the spin-orbit coupling constant. Thus, the perturbed hamiltonian has the form $H=H_{0}+V$, with $V=V_{C F}+V_{S O}$.

The perturbation $V$ mixes the unperturbed $3 d e_{g}$ states with other states and for simplicity it is sufficient to consider only the $4 p$ states $H_{0}\left|p_{\alpha}\right\rangle=E_{p}\left|p_{\alpha}\right\rangle$ with the energy $E_{p}, \alpha=x, y, z$. The two-fold degeneracy is removed in the first order and one can write for one of the perturbed eigenvectors

$$
|\psi\rangle=|0\rangle+\sum_{\alpha} A_{\alpha}\left|p_{\alpha}\right\rangle
$$

where $A_{\alpha}$ are coefficients and $|0\rangle$ is an eigenvector from the subspace spanned by $\left|d_{\gamma}\right\rangle$. The electric dipole moment is given then by

$$
\vec{d}=\langle\psi|e \vec{r}| \psi\rangle=\sum_{\alpha} A_{\alpha}\left\langle 0|e \vec{r}| p_{\alpha}\right\rangle+\text { c.c. }
$$

Assuming $|0\rangle=q_{1}\left|d_{z^{2}}\right\rangle+q_{2}\left|d_{x^{2}-y^{2}}\right\rangle$ we obtain in the first order of perturbation the $z$-component of the electric dipole moment induced by the local crystal field

$$
d_{0 z}=\frac{2 q_{1}^{2} c_{z} e t_{z, z^{2}}^{2}}{\Delta},
$$

where $\Delta=E_{d}-E_{p}$ and $t_{\alpha, \gamma}=\left\langle p_{\alpha}|\alpha| d_{\gamma}\right\rangle$. Due to local $\mathrm{C}_{2}$ crystal field symmetry $d_{0 x}=d_{0 y}=0$. Performing the perturbation up to the third order we get the spinindependent first order contribution and the spin-dependent part of the dipole moment as

$$
\begin{aligned}
& d_{x}=-Q_{x}\left(\frac{\lambda}{\Delta}\right)^{2} S_{x} S_{z}, \\
& d_{y}=-Q_{y}\left(\frac{\lambda}{\Delta}\right)^{2} S_{y} S_{z}, \\
& d_{z}=d_{0 z}+d_{0 z}\left(\frac{\lambda}{\Delta}\right)^{2}\left(S_{x}^{2}+S_{y}^{2}\right),
\end{aligned}
$$

where

$$
Q_{\alpha}=\frac{2 q_{1} c_{z} e t_{z, z^{2}}\left(q_{1} t_{\alpha, z^{2}}+q_{2} t_{\alpha, x^{2}-y^{2}}\right)}{\Delta} .
$$

Thus, in addition to the crystal field induced electric dipole moment the spin-orbit coupling gives rise to spin-dependent contribution.

We now use the obtained results for the above case of magnetic ordering in $\mathrm{MnWO}_{4}$ with $\vec{k}=0$. Each unit cell has two $\mathrm{Mn}^{2+}$ ions in opposite polar surroundings with polar axes along $y$. Therefore, using (5) for both of them and performing the proper cyclic permutation of indices $x, y$ and $z$ we get the electric polarization

$$
\begin{aligned}
& P_{x}=-Q_{x} \frac{1}{2 v}\left(\frac{\lambda}{\Delta}\right)^{2}\left(A_{y} F_{x}+A_{x} F_{y}\right), \\
& P_{y}=d_{0 y} \frac{1}{v}\left(\frac{\lambda}{\Delta}\right)^{2}\left(A_{x} F_{x}+A_{z} F_{z}\right), \\
& P_{z}=-Q_{z} \frac{1}{2 v}\left(\frac{\lambda}{\Delta}\right)^{2}\left(A_{y} F_{z}+A_{z} F_{y}\right),
\end{aligned}
$$


Table 2. Components along $x$ of $\mathrm{Mn}_{1}$ and $\mathrm{Mn}_{2}$ spins induced by $\left(\eta_{1 x}, \xi_{1 x}\right)$ and $\left(\eta_{2 x}, \xi_{2 x}\right)$ in the reference unit cell $\left(0 \cdot \vec{a}_{1}\right)$ and in the unit cell displaced by one period of translation along $a\left(1 \cdot \vec{a}_{1}\right)$. The last two lines show the spin structures in each cell rewritten in terms of $\vec{F}$ and $\vec{A}$.

\begin{tabular}{lll}
\hline & $0 \cdot \vec{a}_{1}$ & $1 \cdot \vec{a}_{1}$ \\
\hline$S_{1 x}$ & $\eta_{1 x}+\eta_{2 x}$ & $\xi_{1 x}+\xi_{2 x}$ \\
$S_{2 x}$ & $-\xi_{1 x}+\xi_{2 x}$ & $\eta_{1 x}-\eta_{2 x}$ \\
\hline$F_{x}$ & $\eta_{1 x}+\eta_{2 x}-\xi_{1 x}+\xi_{2 x}$ & $\eta_{1 x}-\eta_{2 x}+\xi_{1 x}+\xi_{2 x}$ \\
$A_{x}$ & $\eta_{1 x}+\eta_{2 x}+\xi_{1 x}-\xi_{2 x}$ & $-\eta_{1 x}+\eta_{2 x}+\xi_{1 x}+\xi_{2 x}$ \\
\hline
\end{tabular}

where $v$ is the unit cell volume. Therefore, we obtain the same polarization as the one implied by the ME interactions (1). Figure 2d schematically illustrates relations (6) showing $\mathrm{Mn}^{2+}$ ions with spins $\vec{S}_{1}$ and $\vec{S}_{2}$ directed in such a way that the spin-dependent electric dipole moments $\vec{d}_{1}$ and $\vec{d}_{2}$ result in nonzero total dipole moment of the unit cell $\overrightarrow{d_{1}}+\overrightarrow{d_{2}}$.

Other ME interactions present in (11) and absent in (6) can be obtained by performing the quantum perturbations to higher orders. It has to be noted, that when deriving the spin-dependent dipole moments (5) we assumed the polar distortion of the octahedral crystal field to be directed along the $z$ axis (i.e. with two oxygens on the symmetry axis), which is not the case in $\mathrm{MnWO}_{4}$, but this does not change our semiquantitative model.

\section{Magnetic ordering in $\mathrm{MnWO}_{4}$ with $\vec{k}=(1 / 4 ; 1 / 2 ; 1 / 2)$}

Equations (6) give the electric polarization for magnetic structures with $\vec{k}=0$. In $\mathrm{MnWO}_{4}$ the magnetic phase transitions can be described by the order parameters with $\vec{k}=(1 / 4 ; 1 / 2 ; 1 / 2)[16]$. In this point of the Brillouin zone the space group $P 2 / c$ possesses two two-dimensional IR's $G_{1}$ and $G_{2}$. Using the magnetic representation analysis conducted earlier [16], for every direction $\alpha=x, y, z$ we introduce two order parameters $\left(\eta_{1 \alpha}, \xi_{1 \alpha}\right)$ and $\left(\eta_{2 \alpha}, \xi_{2 \alpha}\right)$ transforming according to $G_{1}$ and $G_{2}$, respectively. The spin components along $x$ induced by these order parameters are given in table 2 , For every unit cell one can rewrite the spin components in terms of $\vec{F}$ and $\vec{A}$ as shown in the table. Analogous analysis can be conducted for the $y$ and $z$ spin components.

The magnetic unit cell in $\mathrm{MnWO}_{4}$ is 16 times the crystallographic one. We now sum up $A_{x} F_{x}$ over the magnetic cell $\frac{1}{16} \sum A_{x} F_{x}=2\left(\eta_{1 x} \eta_{2 x}+\xi_{1 x} \xi_{2 x}\right)$ to obtain the electric polarization using (6)

$$
P_{y}=d_{0 y} \frac{2}{v}\left(\frac{\lambda}{\Delta}\right)^{2}\left(\eta_{1 x} \eta_{2 x}+\xi_{1 x} \xi_{2 x}\right) .
$$

Equation (7) gives $P_{y}$ in accordance with the polarization that can be derived from the ME interaction $P_{y}\left(\eta_{1 x} \eta_{2 x}+\xi_{1 x} \xi_{2 x}\right)$ obtained from the macroscopic symmetry analysis [16]. In agreement with the experiment [15] and phenomenological model [16] $P_{y}$ 
arises in the AF2 phase when both $G_{1}$ and $G_{2}$ condense. Long-wavelength modulation does not lead to cancellation of (77) as shown earlier [16]. Similar to (7) other contributions to polarization can be obtained using remaining ME interactions (11).

The numerical value of $P_{y}$ from (7) for $\mathrm{MnWO}_{4}$ can be estimated as follows. We use the real crystal data and oxygen positions from [14] to perform the crystal field expansion (2) and obtain $c_{z} \approx 4.9 \cdot 10^{-9} \mathrm{~N}$ and $v \approx 138 \AA^{3}$. For the matrix elements $t_{\alpha, \gamma}$ we use the hydrogen-like orbitals and obtain $t_{z, z^{2}} \approx 0.67 a_{0} / Z$, where $a_{0}$ is the Bohr radius and $Z$ is the charge of the nucleus and core electrons in units of $e$. Using $Z \approx 5, \lambda \approx 0.05 \mathrm{eV}, \Delta \approx 1 \mathrm{eV}$ and noting that $q_{1} \sim 1,\left(\eta_{1 x} \eta_{2 x}+\xi_{1 x} \xi_{2 x}\right) \sim 1$ we obtain $P_{y} \sim 17 \mu \mathrm{C} / \mathrm{m}^{2}$ in good agreement with the experimental value of the order of $50 \mu \mathrm{C} / \mathrm{m}^{2}$ [15, 17]. In this numerical estimate, however, we considered only one $e_{g}$ level whereas other $3 d$ electrons give comparable contributions to $P_{y}$.

\section{Discussion}

Thus, we have built a microscopic model of ME interactions in magnetoelectrics using $\mathrm{MnWO}_{4}$ as an example. Starting with the hypothetical magnetic order with $\vec{k}=0$ we determined the ME interactions (11). Noting that $\mathrm{Mn}^{2+}$ ions in wolframite are located in noncentrosymmetric polar surroundings we suggested a microscopic model of magnetoelectricity. The orbitals of $3 d$ electrons of $\mathrm{Mn}^{2+}$ ions possess electric dipole moments due to the crystal field influence, which gives rise to additional contributions (such as the considered $4 p$ states) to their wave functions (4). The spinorbit coupling (3) induces spin-dependent electric dipole moments (5) since the angular momentum operator $\vec{L}$ mixes different $\left|p_{\alpha}\right\rangle$ states. In this part our approach combining phenomenological and microscopic models resembles that suggested for the description of weak ferromagnetism by Dzyaloshinskii [18] and Moriya [19]. Our approach differs from those of Sergienko et al. [8] and Katsura et al. [9] who obtain the electric polarization as either $\vec{P} \sim\left[\vec{S}_{i} \times \vec{S}_{i+1}\right]$ or $\vec{P} \sim \vec{e}_{i j} \times\left[\vec{S}_{i} \times \vec{S}_{j}\right]$, which is essentially a result of interacting spins located at two different ions. On the contrary, in our model due to local noncentrosymmetry each magnetic ion has spin-dependent electric dipole moment. We show that for certain spin configurations the sum over all magnetic ions of these spin-dependent electric dipole moments gives rise to macroscopic polarization.

We then apply this approach to describe real magnetic structures in $\mathrm{MnWO}_{4}$ with $\vec{k}=(1 / 4 ; 1 / 2 ; 1 / 2)$ by summing up local contributions to polarization in every unit cell of the magnetic cell. Our approach naturally accounts for the macroscopic symmetry of the unit cell and is valid in both cases of collinear (hypothetical) magnetic ordering with $\vec{k}=0$ and complex long-wavelength modulated magnetic structure observed in $\mathrm{MnWO}_{4}$.

We have chosen $\mathrm{MnWO}_{4}$ as an example since it has $\mathrm{Mn}^{2+}$ ions in polar surroundings

and is directly applicable to our approach. At the same time magnetoelectrics with magnetic ions in noncentrosymmetric environment are numerous and a brief review of the recently discovered multiferroics gives the following examples. Similar to $\mathrm{MnWO}_{4}$ 
the local $\mathrm{C}_{2}$ symmetry is found for $\mathrm{Fe}^{3+}$ ions in $\mathrm{NaFeSi}_{2} \mathrm{O}_{6}$ [20] and for $\mathrm{Ni}^{3+}$ "spine" spins in $\mathrm{Ni}_{3} \mathrm{~V}_{2} \mathrm{O}_{8}$ [21]. Local $\mathrm{C}_{s}$ symmetry is found for $\mathrm{Cu}^{2+}$ ions in $\mathrm{LiCu}_{2} \mathrm{O}_{2}$ [22] and for one of the $\mathrm{Cr}^{3+}$ positions in $\alpha-\mathrm{CaCr}_{2} \mathrm{O}_{4}$ [23]. All of the nonequivalent $\mathrm{Fe}^{3+}$ ions in $\mathrm{FeVO}_{4}$ [24] and $\mathrm{FeTe}_{2} \mathrm{O}_{5} \mathrm{Br}$ [25] have local $\mathrm{C}_{1}$ symmetry. For all these magnetic ions the linear in $\vec{r}$ part of the crystal field expansion can be written as $V_{C F}(\vec{r})=V_{0}+\vec{c} \cdot \vec{r}$ with $|\vec{c}|$ taking values from $9 \cdot 10^{-10} \mathrm{~N}$ for $\mathrm{Ni}_{3} \mathrm{~V}_{2} \mathrm{O}_{8}$ to $6 \cdot 10^{-9} \mathrm{~N}$ for $\mathrm{LiCu}_{2} \mathrm{O}_{2}$.

Magnetoelectric effect was recently found in manganese phosphorus trisulfide $\mathrm{MnPS}_{3}$ [26]. $\mathrm{MnPS}_{3}$ possesses a monoclinic crystal structure $C 2 / m$ and shows an antiferromagnetic collinear order with $\vec{k}=0$ below $\mathrm{T}_{N}=78 \mathrm{~K}$. Similar to $\mathrm{MnWO}_{4}$ it has two $\mathrm{Mn}^{2+}$ ions in the unit cell with the local $\mathrm{C}_{2}$ symmetry and our analysis performed above for $\vec{k}=0$ magnetic structures in wolframite is directly applicable to $\mathrm{MnPS}_{3}$. According to the neutron diffraction data the magnetic structure is characterized by $A_{x}$ and $A_{z} \neq 0$ below $\mathrm{T}_{N}$. Thus, following the Eqs. (1) one can expect linear magnetoelectric effect below $\mathrm{T}_{N}$ with magnetoelectric interactions $P_{\mu} A_{x} F_{y}, P_{\mu} A_{z} F_{y}, P_{y} A_{\mu} F_{\mu}, P_{y} A_{x} F_{z}$ and $P_{y} A_{z} F_{x}$, where $\mu=x, z$. According to Eqs. (6) the magnetoelectric coefficient $\alpha_{y z}=\mathrm{d} P_{y} / \mathrm{d} H_{z}$, for example, can be estimated as $\alpha_{y z}=d_{0 z}(1 / v)(\lambda / \Delta)^{2} A_{z} \cdot \mathrm{d} F_{z} / \mathrm{d} H_{z}$, with $\mathrm{d} F_{z} / \mathrm{d} H_{z} \sim 2 \cdot 10^{-6} \mathrm{Oe}^{-1}[27]$ and $A_{z} \sim 1$. The polar local distortion of the $\mathrm{Mn}^{2+}$ environment in $\mathrm{MnPS}_{3}$ is much smaller than in wolframite giving $c_{z} \approx 1.4 \cdot 10^{-11} \mathrm{~N}$. Using $v \approx 207 \AA^{3}$ we obtain $\alpha_{y z} \sim 3.4 \cdot 10^{-8} \mu \mathrm{C} \cdot \mathrm{m}^{-2} \cdot \mathrm{Oe}^{-1}$, which is rather small.

$\mathrm{LiNiPO}_{4}$ possesses an orthorhombic symmetry with space group Pnma and shows linear magnetoelectric effect in the low temperature $C$-type commensurate antiferromagnetic phase with $\vec{k}=0$ below $20.8 \mathrm{~K}$ [28, 29]. The spins are predominately directed along the $c$ axis and the magnetic structure is described by the order parameter $C_{z}$ transforming according to the IR $\mathrm{GM}^{4-}$. The phenomenological magnetoelectric interactions $C_{z} M_{z} P_{x}$ and $C_{z} M_{x} P_{z}$ were suggested earlier [28] and here we can estimate the magnetoelectric coefficient according to our microscopic model. Our microscopic approach differs from that suggested for $\mathrm{LiNiPO}_{4}$ earlier [30], which is based on lowering the superexchange interaction energy due to the uniform displacement of oxygen tetrahedra. The local symmetry of the $\mathrm{Ni}^{2+}$ ions surroundings is $\mathrm{C}_{s}$ with $|\vec{c}| \approx 4.3 \cdot 10^{-9} \mathrm{~N}$. Similar to the above case of $\mathrm{MnPS}_{3}$ using $\mathrm{d} M_{x} / \mathrm{d} H_{x} \sim 2 \cdot 10^{-2} \mu_{\mathrm{B}} / \mathrm{T}$ per Ni-atom [29] we obtain an estimation of the value of magnetoelectric coefficient $\alpha_{z x} \approx$ $0.31 \mu \mathrm{C} \cdot \mathrm{m}^{-2} \cdot \mathrm{T}^{-1}$ in good agreement with the experimental value of $0.2 \mu \mathrm{C} \cdot \mathrm{m}^{-2} \cdot \mathrm{T}^{-1}[28]$ and about three orders of magnitude higher than that in $\mathrm{MnPS}_{3}$.

At the same time in many other magnetoelectrics magnetic ions are located in centrosymmetric positions. Such is the case, for example, in the rare-earth manganites $\mathrm{RMnO}_{3}$ [31] and $\mathrm{CuO}$ [32] where the local symmetry around $\mathrm{Mn}^{3+}$ and $\mathrm{Cu}^{2+}$ is $\mathrm{C}_{i}$. Nevertheless, our approach is valid also in these cases. The application of our model becomes more complicated and will be published elsewhere [33], but briefly can be described as follows. The local symmetry around the rare-earth ions in $\mathrm{RMnO}_{3}$ is $\mathrm{C}_{s}$. The importance of rare-earth ions in formation of electric polarization in $\mathrm{RMnO}_{3}$ was recently pointed out [34, 35]. Indeed, the magnetic order of $\mathrm{Mn}^{3+}$ ions induces magnetic ordering of the rare-earths through various exchange mechanisms, which makes 
our approach applicable. In the case of $\mathrm{CuO}$ the oxygens have local symmetry $\mathrm{C}_{2}$. The role of oxygen in the superexchange is still a subject of debate [11, 36]. Being the intermediate ion conducting superexchange, $\mathrm{O}^{2-}$ should possess induced magnetic moment when copper spins order, which again allows application of our model. The antiferromagnetic spin polarization at the oxygen sites was measured, for example, in the multiferroic $\mathrm{TbMn}_{2} \mathrm{O}_{5}$ [37].

\section{Conclusions}

We have suggested a microscopic model of magnetoelectric interactions, which directly exploits the fact that in many magnetoelectrics magnetic ions are located in noncentrosymmetric positions. The model is illustrated by the examples of $\mathrm{MnWO}_{4}$ and $\mathrm{LiNiPO}_{4}$, for which we obtained good correspondence of the values of electric polarization and magnetoelectric coefficient, respectively. We also give an estimate of the magnetoelectric coefficient in $\mathrm{MnPS}_{3}$.

\section{References}

[1] Curie P. J. Physique, 3:393, 1894.

[2] Dzyaloshinskii I E. Sov. Phys.-JETP, 10:628, 1959.

[3] Astrov D N. Sov. Phys.-JETP, 11:708, 1960.

[4] Tokura Y and Seki S. Adv. Mater., 22:1554, 2010.

[5] Arima T. J. Phys. Soc. Jap., 80:052001, 2011.

[6] Sakhnenko V P and Ter-Oganessian N V. Ferroelectrics, 400:12, 2010.

[7] Kimura H, Kobayashi S, Wakimoto S, Noda Y, and Kohn K. Ferroelectrics, 354:77, 2007.

[8] Sergienko I A and Dagotto E. Phys. Rev. B, 73:094434, 2006.

[9] Katsura H, Nagaosa N, and Balatsky A V. Phys. Rev. Lett., 95:057205, 2005.

[10] Mochizuki M and Furukawa N. Phys. Rev. Lett., 105:187601, 2010.

[11] Moskvin A S and Drechsler S-L. Phys. Rev. B, 78:024102, 2008.

[12] Moskvin A S and Drechsler S-L. Eur. Phys. J. B, 71:331, 2009.

[13] Jia C, Onoda S, Nagaosa N, and Han J H. Phys. Rev. B, 74:224444, 2006.

[14] Lautenschläger G, Weitzel H, Vogt T, Hock R, Bohm A, Bonnet M, and Fuess H. Phys. Rev. B, 48:6087, 1993.

[15] Taniguchi K, Abe N, Takenobu T, Iwasa Y, and Arima T. Phys. Rev. Lett., 97:097203, 2006.

[16] Sakhnenko V P and Ter-Oganessian N V. J. Phys.: Condens. Matter, 22:226002, 2010.

[17] Arkenbout A H, Palstra T T M, Siegrist T, and Kimura T. Phys. Rev. B, 74:184431, 2006.

[18] Dzyaloshinskii I. J. Phys. Chem. Solids, 4:241, 1958.

[19] Moriya T. Phys. Rev., 120:91, 1960.

[20] Jodlauk S, Becker P, Mydosh J A, Khomskii D I, Lorenz T, Streltsov S V, Hezel D C, and Bohatý L. J. Phys.: Condens. Matter, 19:432201, 2007.

[21] Lawes G, Harris A B, Kimura T, Rogado N, Cava R J, Aharony A, Entin-Wohlman O, Yildirim T, Kenzelmann M, Broholm C, and Ramirez A P. Phys. Rev. Lett., 95:087205, 2005.

[22] Park S, Choi Y J, Zhang C L, and Cheong S-W. Phys. Rev. Lett., 98:057601, 2007.

[23] Singh K, Simon C, and Toledano P. Phys. Rev. B, 84:064129, 2011.

[24] Dixit A and Lawes G. J. Phys.: Condens. Matter, 21:456003, 2009.

[25] Zaharko O, Pregelj M, Arčon D, Brown P J, Chernyshov D, Stuhr U, and Berger H. J. Phys.: Conf. Ser., 211:012002, 2010. 
[26] Ressouche E, Loire M, Simonet V, Ballou R, Stunault A, and Wildes A. Phys. Rev. B, $82: 100408(\mathrm{R}), 2010$.

[27] Toyoshima W, Masubuchi T, Watanabe T, Takase K, Matsubayashi K, Uwatoko Y, and Takano Y. J. Phys.: Conf. Ser., 150:042215, 2009.

[28] Kornev I, Bichurin M, Rivera J-P, Gentil S, Schmid H, Jansen A G M, and Wyder P. Phys. Rev. $B, 62: 12247,2000$.

[29] Toft-Petersen R, Jensen J, Jensen T B S, Andersen N H, Christensen N B, Niedermayer C, Kenzelmann M, Skoulatos M, Le M D, Lefmann K, Hansen S R, Li J, Zarestky J L, and Vaknin D. Phys. Rev. B, 84:054408, 2011.

[30] Jensen T B S, Christensen N B, Kenzelmann M, Rønnow H M, Niedermayer C, Andersen N H, Lefmann K, Schefer J, Zimmermann M v, Li J, Zarestky J L, and Vaknin D. Phys. Rev. B, 79:092412, 2009.

[31] Kimura T, Lawes G, Goto T, Tokura Y, and Ramirez A P. Phys. Rev. B, 71:224425, 2005.

[32] Kimura T, Sekio Y, Nakamura H, Siegrist T, and Ramirez A P. Nat. Mater., 7:291, 2008.

[33] Sakhnenko V P and Ter-Oganessian N V. To be published.

[34] Feyerherm R, Dudzik E, Prokhnenko O, and Argyriou D N. J. Phys.: Conf. Ser., 200:012032, 2010.

[35] Schierle E, Soltwisch V, Schmitz D, Feyerherm R, Maljuk A, Yokaichiya F, Argyriou D N, and Weschke E. Phys. Rev. Lett., 105:167207, 2010.

[36] Moskvin A S. JETP, 104:913, 2007.

[37] Beale T A W, Wilkins S B, Johnson R D, Bland S R, Joly Y, Forrest T R, McMorrow D F, Yakhou F, Prabhakaran D, Boothroyd A T, and Hatton P D. Phys. Rev. Lett., 105:087203, 2010. 